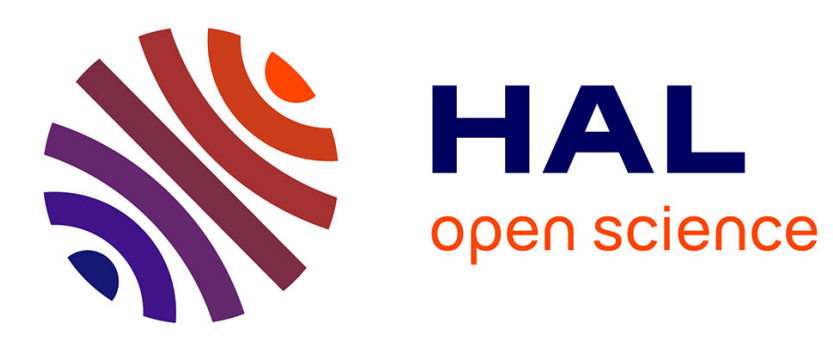

\title{
Detecting a Zeptogram of Pyridine with a Hybrid Plasmonic-Photonic Nanosensor
}

Julien Proust, Jérôme Martin, Davy Gérard, Jean-Louis Bijeon, Jerome Plain

\section{To cite this version:}

Julien Proust, Jérôme Martin, Davy Gérard, Jean-Louis Bijeon, Jerome Plain. Detecting a Zeptogram of Pyridine with a Hybrid Plasmonic-Photonic Nanosensor. ACS Sensors, 2019, 4 (3), pp.586-594. 10.1021/acssensors.8b01068. hal-02301706

\section{HAL Id: hal-02301706 \\ https://hal-utt.archives-ouvertes.fr/hal-02301706}

Submitted on 21 May 2021

HAL is a multi-disciplinary open access archive for the deposit and dissemination of scientific research documents, whether they are published or not. The documents may come from teaching and research institutions in France or abroad, or from public or private research centers.
L'archive ouverte pluridisciplinaire HAL, est destinée au dépôt et à la diffusion de documents scientifiques de niveau recherche, publiés ou non, émanant des établissements d'enseignement et de recherche français ou étrangers, des laboratoires publics ou privés. 


\title{
Detecting a zeptogram of pyridine with a hybrid plasmonic-photonic nanosensor
}

Julien Proust, ${ }^{*}$ Jérôme Martin, Davy Gérard, Jean-Louis Bijeon, and Jérôme Plain*

Light, Nanomaterials, Nanotechnologies (L2n), Institut Charles Delaunay, CNRS, Université de Technologie de Troyes, France

E-mail: julien.proust@utt.fr; jerome.plain@utt.fr

\begin{abstract}
Thanks to their small sensing volume, nanosensors based on localized surface plasmon resonances (LSPR) allow the detection of minute amounts of analytes, down to the single molecule limit. However, the detected analytes are often large molecules, such as proteins. The detection of small molecules remains largely unexplored. Here, we use a hybrid photonic-plasmonic nanosensor to detect a small target molecule (pyridine). The sensor's design is based on a dielectric photonic microstructure acting as an antenna, which efficiently funnels light towards a plasmonic transducer and enhance the detection efficiency. This sensor exhibits a limit of detection as small as $10^{-14}$ mol. $L^{-1}$. Using a calibration procedure based on electrodynamical numerical simulations, we compute the number of detected molecules. This yields a limit of detection in mass of 4 zeptograms $\left(1 \mathrm{zg}=10^{-21} \mathrm{~g}\right)$, a record value for plasmonic molecular sensors. Our system can hence be seen as an optical molecular weighing scale, enabling room temperature detection of mass at the zeptogram scale.
\end{abstract}




\section{Keywords}

Nanosensors, surface plasmons, optical antennas, molecular weighing scales, pyridine, limit of detection

Plasmonic sensors are optical refractometric sensors using noble metal nanostructures as their sensing unit, or transducer. ${ }^{1,2}$ Two kinds of plasmonic sensors can be distinguished, depending on the nature of the plasmonic resonance hosted by the metallic structure. First, thin metallic films support a surface wave called a surface plasmon resonance (SPR), propagating along the surface, which is extremely sensitive to the refractive index of the neighboring medium. The SPR geometry offers a simple platform for label-free biosensing applications and is now used in routine analytical instruments. ${ }^{3,4}$ The second approach relies on metallic nanostructures exhibiting localized surface plasmon resonances (LSPR). ${ }^{5}$ In contrast with the SPR, the LSPR is fully confined around the nanostructure, yielding an extremely small interaction (i.e. sensing) volume. The spectral position of the LSPR depends notably on the refractive index of the medium located inside this sensing volume. Due to the nanometric size of their transducer, LSPR sensors exhibit a number of advantages when compared to SPR sensors: (i) smaller amounts of analytes are required, (ii) parallel analysis is possible enabling high-throughput capabilities and (iii) the potential for miniaturization is high. ${ }^{6}$ However, the small transducer size turns into a problem when it comes to the optical monitoring of the LSPR: addressing a single nanoparticle smaller than $100 \mathrm{~nm}$ with a beam of light either deteriorates the signal-to-noise ratio on the detection channel, or requires a complex optical setup with expansive objective lenses. This explains why, in spite of the tremendous amount of papers demonstrating the capabilities of LSPR sensors, they remain mainly proof-of-concept laboratory objects.

In order to selectively and efficiently address a single nanoparticle with light, it is possible to use optical antennas. Optical antennas are devices converting an incident propagating wave into a localized excitation, and vice-versa. ${ }^{7}$ Of particular interest are dielectric antennas, ${ }^{8}$ as dielectric materials exhibit very low losses and are often biocompatible. For instance, 
dielectric microspheres can focus light into nanometric volumes and enhance the collection of fluorescence from single molecules. ${ }^{9}$ In a previous work, ${ }^{10}$ we showed that quasi-axicon lenses consisting of glass micropyramids were able to generate intense Bessel-like beams. Bessel beams are low-divergence light beams whose intensity profile can be described by a Bessel function. We demonstrated that the uniform propagation length of the light is six times longer for the quasi-Bessel beam exiting the micropyramid than for a Gaussian beam.

In this paper, we propose a hybrid plasmonic nanosensor based on a metallic nanostructure sitting on the apex of a glass micropyramid. The micropyramid acts as an axicon lens, allowing for an efficient excitation of the transducer from weakly focused impinging light, as well as an efficient collection of the transmitted light due to the high directivity of the quasi-Bessel beam. Micropyramids are fabricated over a large scale $\left(1 \mathrm{~cm}^{2}\right)$ using optical interferometric lithography and wet etching. This simple approach makes possible the realization of an array of hybrid nanosensors, a key for high throughput parallel analysis. The optical transducer at the apex of the micropyramid is either a single gold nanoparticle (monomer) or a pair of coupled gold nanoparticles (dimer). We demonstrate, using a small target molecule (pyridine), that minute amounts of analytes can be detected. Furthermore, we introduce a calibration procedure relying on electrodynamical numerical simulations that allows us to retrieve the number of pyridine molecules, and hence the molecular mass, adsorbed on the sensor. A limit of detection of a few zeptograms is evidenced.

\section{Design and fabrication}

Figure 1a shows the principle of the hybrid sensor. It consists of two complementary parts. The first one is a dielectric optical antenna based on a glass micropyramid. ${ }^{10}$ The second one is the plasmonic transducer consisting of one or two gold nanoparticles (GNP) sitting on the apex of the lens. This design allows for a significant enhancement of both the excitation intensity at the gold nanoparticle position and the detection efficiency. The fabrication 

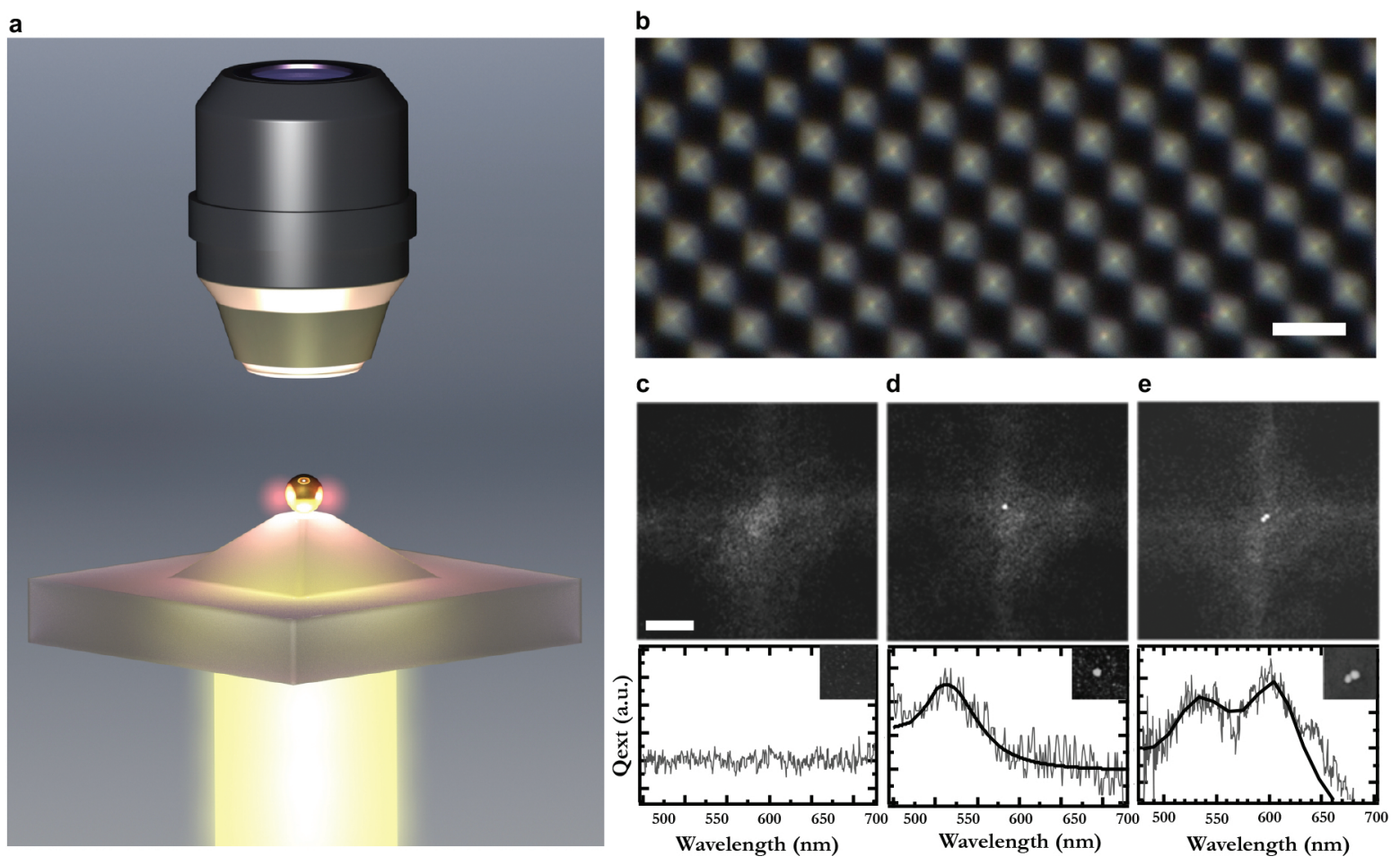

Figure 1: a) Principle of the hybrid sensor: a quasi-axicon glass lens (micropyramid) with a single GNP on top. b) Dark-field image of an array of quasi-axicon lenses (scale bar $4 \mu \mathrm{m}$ ). c-e) SEM images (scale bar $500 \mathrm{~nm}$ ) of quasi-axicon lens with c) zero, d) one and e) two gold nanoparticles on top, and their corresponding extinction spectrum. The experimental spectra (gray curves) are compared with d) Mie theory for a single sphere of $50 \mathrm{~nm}$, and e) DDA calculations for two spheres of $50 \mathrm{~nm}$ with a gap of $0.7 \mathrm{~nm}$ (thick black curves).

method is based on a combination of chemical functionalization, optical lithography and wet etching (see Experimental Section). Figure 1b shows a typical dark field image of an array of hybrid photonic-plasmonic sensors. It is a square array with a period $\mathrm{P}=4 \mu \mathrm{m}$, covering an area of about $1 \mathrm{~cm}^{2}$. A detailed morphological characterization with atomic force microscopy has been performed and is shown in the Supplementary Information (Figure S1). The distance between each sensor is sufficiently high to measure the optical response from a single transducer. In order to further characterize our array, Figures 1c-e present SEM images and the corresponding extinction spectra for a tip without nanoparticle (c), with a gold monomer with a diameter of $50 \mathrm{~nm}(\mathrm{~d})$, and a gold dimer constituted of two particles with a diameter of $50 \mathrm{~nm}$ (e). Both spectra have been recorded under unpolarized light 
excitation (see Experimental Section for details). The single peak observed in the monomer spectra is the signature of the dipolar LSPR of the GNP. The two peaks visible in the dimer spectra are linked to the two LSPR modes associated with the two polarizations of the excitation (transverse and longitudinal).

We measure an enhancement of one order of magnitude of the optical detected signal to noise ratio (SNR) with the quasi-axicon lens compared to a flat substrate. The measured SNR (up to $13.6 \mathrm{~dB}$, see Figure S2) is directly comparable to the SNR obtained with a dedicated confocal microscope $\operatorname{setup}^{11}(13.3 \mathrm{~dB})$. This demonstrates the capability of the glass micropyramid to act as an efficient optical antenna. In order to prove that we retrieve the extinction spectrum of the GNP sitting at the apex of the quasi-axicon lens, we performed numerical simulations of the GNP plasmonic response. The spectrum of the single GNP (Figure 1d) has been compared with the result of Mie theory for a $50 \mathrm{~nm}$ diameter gold

sphere. A very good agreement is observed, evidencing the small diameter dispersion of our chemically synthesized GNPs. In the case of the gold dimer, we used the Discrete Dipole Approximation (DDA) method for two $50 \mathrm{~nm}$ gold spheres with a varying gap distance. A good agreement between the calculation and the experiment is observed for a gap distance of $0.7 \mathrm{~nm}$ (Figure 1e). Thus, we are able to characterize the distance between the two coupled GNPs using the extinction spectrum as a plasmonic ruler.

\section{Sensing experiment}

\section{Choice of the analyte}

The choice of the analyte is an essential point for sensing experiments. We choose pyridine (CAS number 110-86-1) for this study as the target molecule. Three reasons motivate our choice.

(i) Pyridine is an aromatic molecule exhibiting different contributing structures. Its most stable form is presented in Figure S3a and exhibits an electric dipole moment. This 
configuration allows a packed stacking of pyridine on gold, ${ }^{12}$ as presented in Figures S3b-c. This important fact helped us to determine the number of adsorbed molecules, assuming a dense stacking onto the gold surface.

(ii) Pyridine presents a high chemical affinity with gold, ensuring that the molecules will be attracted towards the GNP.

(iii) It has been demonstrated that the surfactant layer (sodium citrate molecules) located at the GNP surface desorbs in presence of pyridine. ${ }^{13}$ As a consequence, we can safely assume that no more sodium citrate molecules remained on the GNP surface during our experiments.

\section{Measurements}

Sensing measurements were performed following the sequence shown in Figure 2. A droplet of a low concentration $\left(10^{-15}\right.$ mol. $\left.\mathrm{L}^{-1}\right)$ solution of pyridine diluted in 2-propanol (IPA) was deposited on the structured substrate. The high wettability of IPA on glass allowed a homogenous dispersion of pyridine over the substrate. We emphasize that the drying of the solution is not uniform due to the structuration of the substrate. We observed that the solution escaped the unstructured areas to concentrate on the areas with micropyramids (see the optical image in the inset of Figure 2, step 4). With evaporation, droplets were formed around each micropyramid, finally leading to the adsorption of pyridine on the GNPs thanks to the high chemical affinity of pyridine with gold. The overall result of the evaporation process is a concentration of the molecules onto the transducer. A similar process was previously reported in more complex structured nanosensors consisting of an array of silicon micropillars with metallic nanotips on their top. ${ }^{14}$

Optical characterization was then performed by extinction spectroscopy using a homemade confocal microscope. The micropyramids were illuminated from the bottom with slightly focused unpolarized white light (Figure 1a). The confocal microscope collects most 


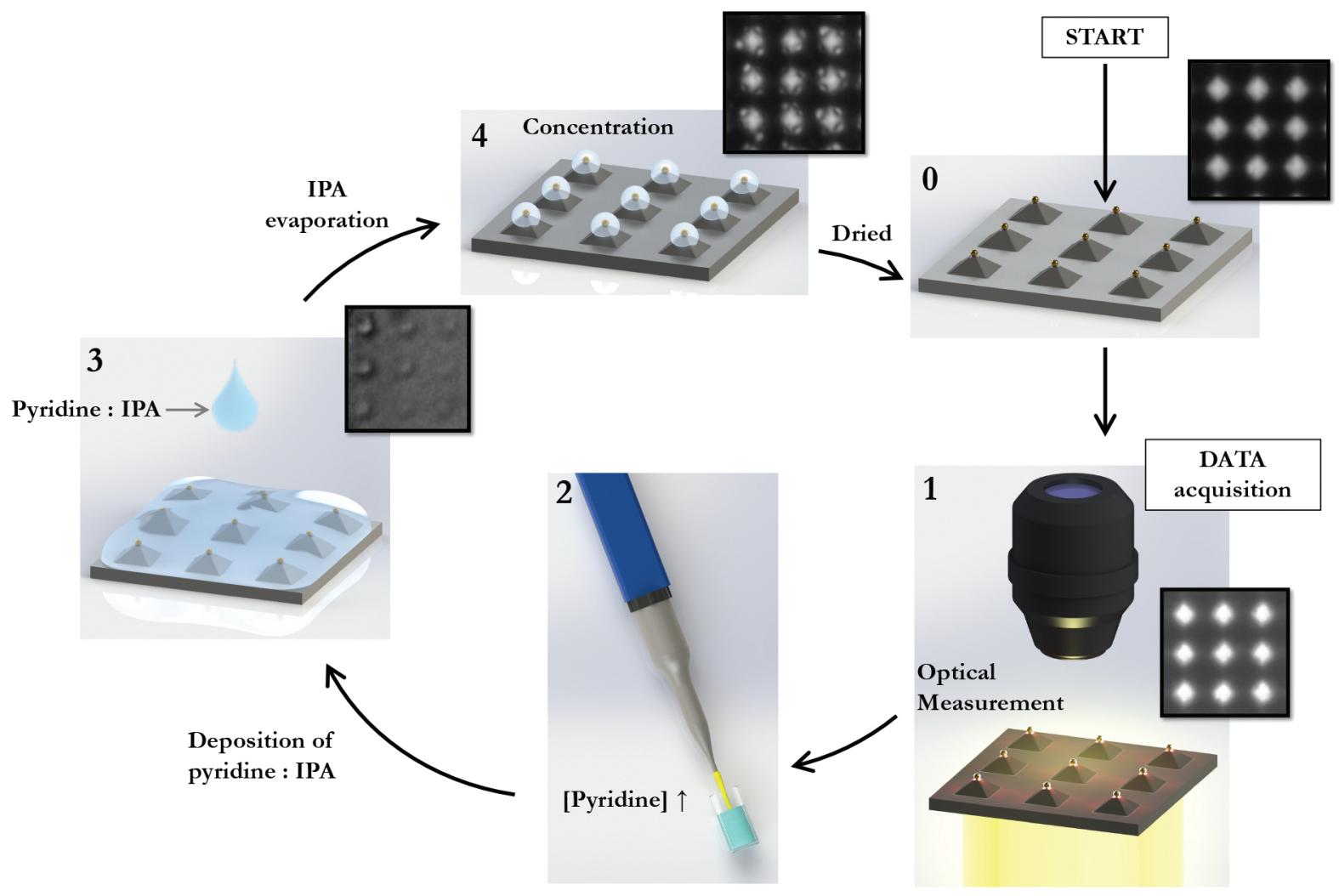

Figure 2: Sensing procedure. Step 0: pristine sample. Step 1: Optical measurement. Step 2: $100 \mu \mathrm{L}$ of pyridine is taken. The concentration is increased 5-fold each cycle, starting from $10^{-15}$ mol.L ${ }^{-1}$. Step 3: Pyridine is deposited on the sample. Step 4: The evaporation of the solvent concentrates the pyridine molecules on the axicon lenses. The cycle starts again with a new optical measurement. Insets: optical images $\left(15 \times 15 \mu \mathrm{m}^{2}\right)$ corresponding to the different steps. 

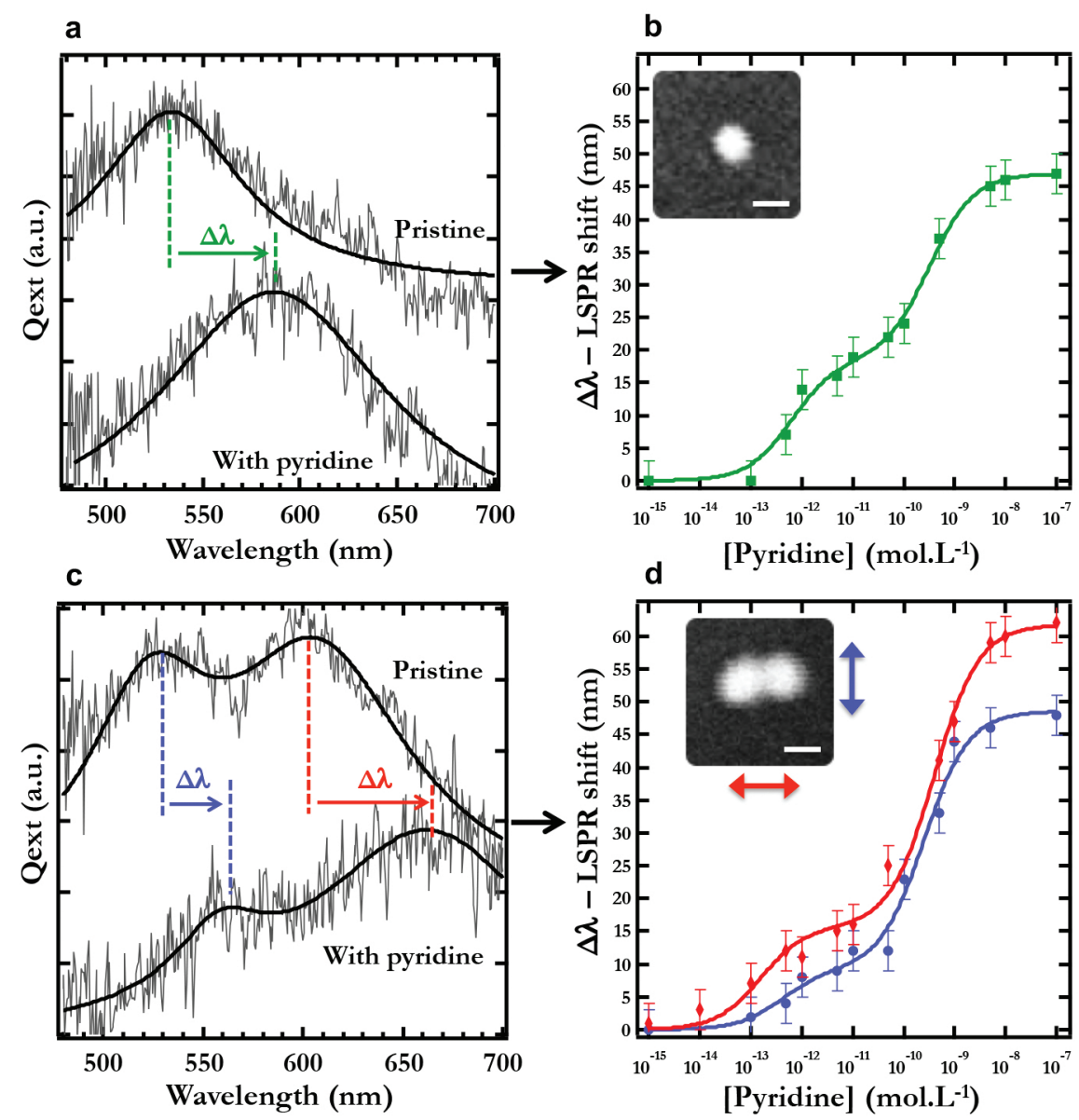

Figure 3: a) Raw data from the optical measurement on gold monomer: pristine sensor (upper curve) and sensor exposed to a pyridine concentration of $10^{-7}$ mol.L ${ }^{-1}$ (bottom curve). The thick black line is the result of a smoothing of the raw data. b) Plot of the LSPR shift $\Delta \lambda$ as a function of the pyridine concentration. Inset: SEM image of the sensor. c) Same as (a) for a gold dimer. d) Plot of the LSPR shifts for longitudinal (red curve) and transverse (blue curve) modes plotted as a function of the pyridine concentration. Inset: SEM images, scale bar is $50 \mathrm{~nm}$. 
of the light exiting from a single micropyramid (after interaction with the transducer). After the measurement at a given concentration, a new droplet of the pyridine solution with a 5-fold higher concentration was deposited onto the substrate, followed by evaporation and a new optical measurement. We repeated this process for pyridine concentrations ranging from $10^{-15}$ mol.L $\mathrm{L}^{-1}$ to $10^{-7} \mathrm{~mol} \cdot \mathrm{L}^{-1}$.

Extinction spectra of two representative micropyramids are shown in Figure 3a,c: one decorated with a single gold nanoparticle; and one decorated with a gold dimer (see SEM images in Figure 3b,d, insets). The extinction spectra for both sensors are shown for two different cases: first before pyridine deposition (pristine sensor) and then after the deposition of pyridine at $10^{-7}$ mol.L $\mathrm{L}^{-1}$. For each sensor, a clear red-shift of the LSPR is observed after pyridine deposition. The observed red shift of the resonance, denoted $\Delta \lambda$, is a well-known phenomenon. ${ }^{15}$ The accumulation of molecules around the gold nanoparticles (in the nearfield) increases the effective optical index of the surrounding medium. The refractive index of liquid pyridine is $1.507\left(\lambda=589.3 \mathrm{~nm}^{16}\right)$ and can increase during crystallization. ${ }^{17}$ The LSPR position is directly linked to the refractive index.

The resonance shift $\Delta \lambda$ is plotted in Figure $3 \mathrm{~b}$ and $\mathrm{e}$ as a function of the pyridine concentration for the monomer and the two polarizations of the dimer, respectively. We observe an increasing shift with the pyridine concentration. The first measurable red-shift appears at a concentration of $5.10^{-13}$ mol.L $\mathrm{L}^{-1}$ for both the monomer and the transversal mode of the dimer. For the longitudinal mode of the dimer it appears at $10^{-14} \mathrm{~mol}^{-1} \mathrm{~L}^{-1}$, evidencing a better limit of detection (LoD). At high concentration, each curve presents a plateau associated with the saturation of the sensor. This behavior is well known for plasmonic sensors and stems from the limited extension of the optical near-field around the GNP. Interestingly, another plateau is observed for lower concentrations around $\Delta \lambda \approx 10-15$ nm. As a consequence, we model the sensor's experimental response using a double Langmuir isotherm adapted from: ${ }^{18}$ 


$$
\Delta \lambda=\frac{\Delta \lambda_{1} \cdot K_{1} \cdot[\text { Pyridine }]}{1+K_{1} \cdot[\text { Pyridine }]}+\frac{\Delta \lambda_{2} \cdot K_{2} \cdot[\text { Pyridine }]}{1+K_{2} \cdot[\text { Pyridine }]}
$$

where $\Delta \lambda_{1}$ and $\Delta \lambda_{2}$ are the maximum resonance shifts associated to the two different isotherms, $K_{1}$ and $K_{2}$ are two thermodynamic affinity constants, and [Pyridine] is the pyridine concentration. Eq. 1 was used to fit the experimental data. Results are shown as solid lines in Figure 3b and d. A good agreement is observed.

Table 1: Fitting parameters of the double Langmuir isotherms using Eq. 1.

\begin{tabular}{|l||c|c|c|c|}
\hline & $\Delta \lambda_{1}(\mathrm{~nm})$ & $K_{1}\left(10^{12} \cdot \mathrm{mol}^{-1}\right)$ & $\Delta \lambda_{2}(\mathrm{~nm})$ & $K_{2}\left(10^{9} \cdot \mathrm{mol}^{-1}\right)$ \\
\hline \hline Monomer & $18.5 \pm 1.4$ & $1.6 \pm 0.14$ & $28.5 \pm 1.6$ & $3.2 \pm 0.82$ \\
\hline Dimer (transversal) & $9.3 \pm 0.3$ & $2.4 \pm 0.32$ & $39.3 \pm 0.3$ & $4.0 \pm 0.15$ \\
\hline Dimer (longitudinal) & $15.7 \pm 0.2$ & $6.4 \pm 0.52$ & $46.1 \pm 0.3$ & $2.5 \pm 0.07$ \\
\hline
\end{tabular}

We point out that the monomer and the transversal mode of the dimer exhibit very similar sensing behaviors, while the longitudinal mode of the dimer has a better limit of detection and a higher maximum shift. Table 1 explains in details the parameters corresponding to the two regions of the fit from Eq. 1. We observe that the first isotherm corresponds to a red shift between 9.3 and $18.5 \mathrm{~nm}$, while the second one between 28.5 and $46.1 \mathrm{~nm}$. These two red shifts correspond to two different phenomena, as detailed below. In the following, we denote Region 1 for the zone with the first red-shift and Region 2 for the zone corresponding to the larger red-shift. Let us emphasize that a similar sensing behavior (two plateaus) has been observed previously. ${ }^{19,20}$

\section{Discussion}

\section{Modeling and calibration}

To understand the different mechanisms leading to the sensor's optical response, it is important to quantify the number of pyridine molecules adsorbed on the gold nanoparticles. 
An estimate of this number can be obtained by using the fingerprint of the pyridine linked by a nitrogen atom on gold $\left(0.16 \mathrm{~nm}^{2}\right.$, estimated from the density of crystallized pyridine $\mathrm{e}^{21}$ and STM images of aromatic molecules ${ }^{22}$ ) and considering a compact staking on the gold free surface. For a single $50 \mathrm{~nm}$ spherical GNP, this yields to $\sim 50000$ molecules in order to form the first monolayer. The spatial extension of the optical near-field around a $50 \mathrm{~nm}$ GNP is $\approx 25 \mathrm{~nm} .{ }^{23}$ To entirely fill the associated volume, $3.4 \times 10^{6}$ molecules are required. In the following, we use electromagnetic numerical computations to correlate the pyridine concentration in the droplet to the number of molecules adsorbed on the transducer.

Using a FDTD commercial solution (OptiFDTD, from Optiwave), we computed the extinction spectra of a monomer and a dimer for various thickness of a close packed arrangement of pyridine. The considered geometry for such calculations is shown in Figure 4a. We considered a plane wave illumination and a flat substrate. Calculations taking into account the pyramidal shape (not shown) have also been performed and did not show any significant deviation from the flat surface case. The pyridine molecules are considered to be arranged into a close packed form. As a consequence, we consider the adsorbed molecules to be a homogeneous dielectric shell $(\mathrm{n}=1.507)$ surrounding the GNP. To simulate the quantity of pyridine around the sensor, we tune the thickness of the dielectric shell (Supplementary Information, Figure S3c). Figures $4 \mathrm{~b}$ and $4 \mathrm{c}$ show the calculated spectra respectively for the monomer and the dimer with different thicknesses of pyridine. As observed experimentally, the maximum of the plasmon resonance is red-shifted with the increase of the shell thickness $d$ (i.e. an increase of the effective refractive index probed by the GNP). As a consequence, it is possible to associate with each observed resonance shift $\Delta \lambda$ a corresponding pyridine shell thickness. Dividing the volume of the shell by the crystal pyridine volume yields to an estimation of the number of pyridine molecules adsorbed onto the sensor. Figure 4d depicts a plot of the resonance shift $\Delta \lambda$ as a function of the number of pyridine molecules adsorbed onto the monomer, while Figures $4 \mathrm{e}$ and $4 \mathrm{f}$ correspond to the dimer case for transverse and longitudinal polarizations, respectively. FDTD calculations (pink triangles) are 
a

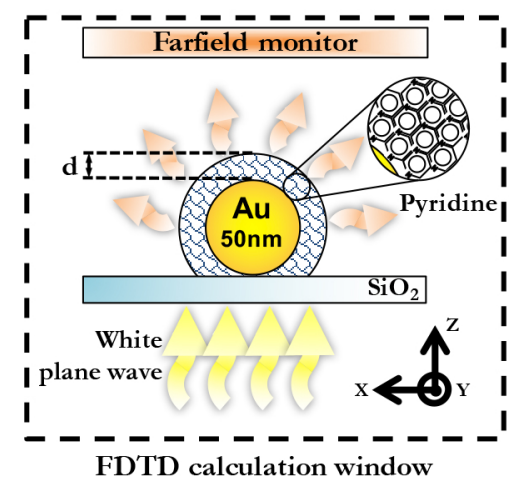

b

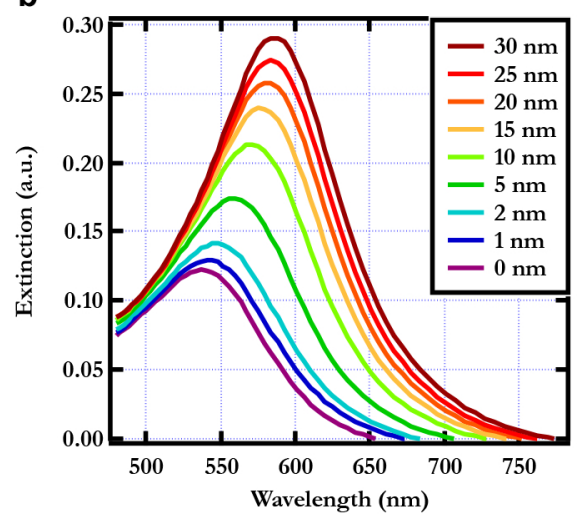

C

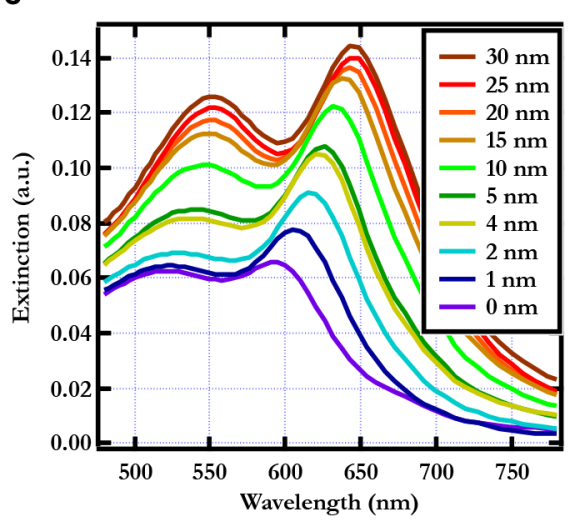

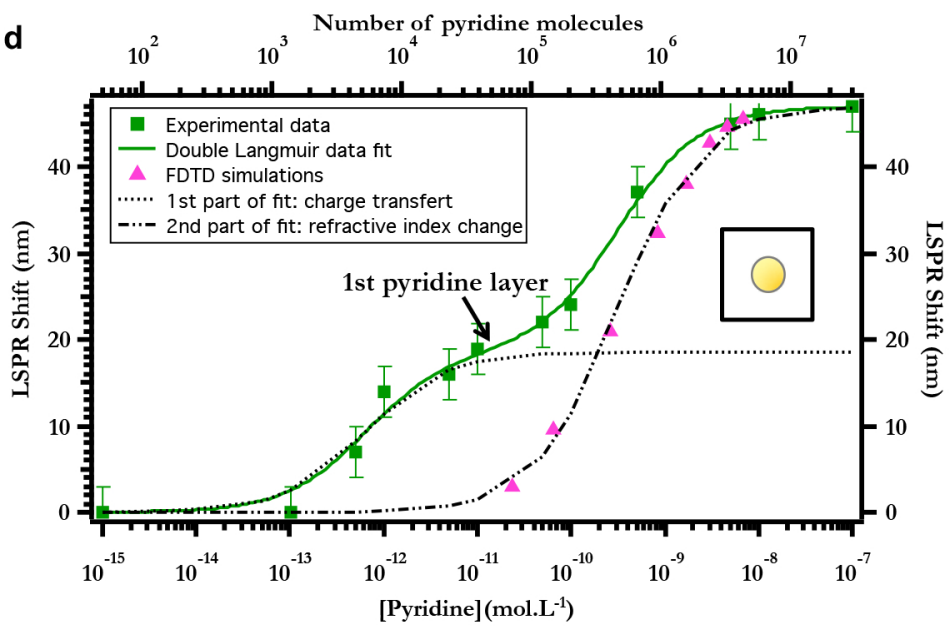
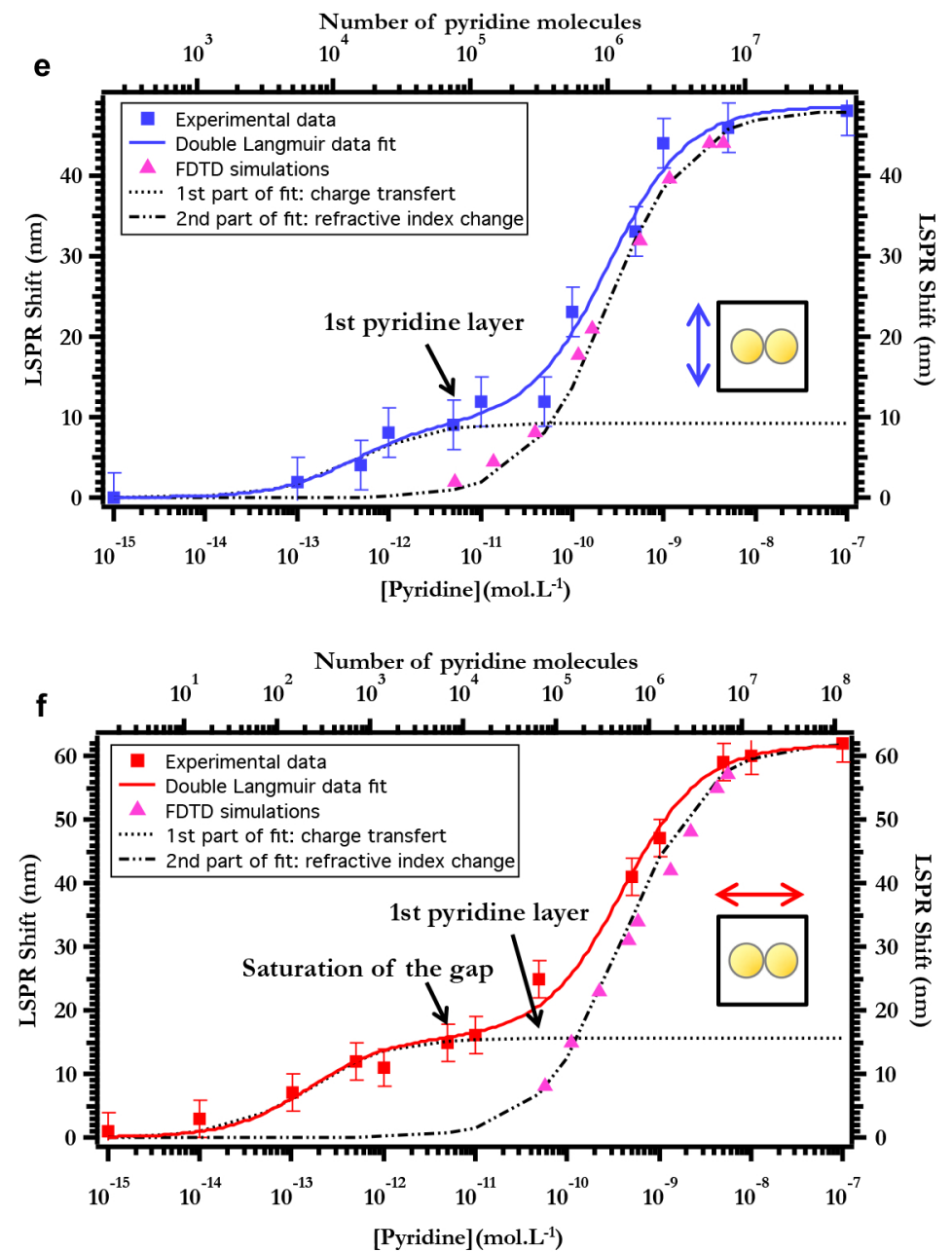

Figure 4: a) Principle of the FDTD calculation. b) FDTD calculated extinction spectra for a $50 \mathrm{~nm}$ monomer. c) FDTD calculated extinction spectra for a $2 \times 50 \mathrm{~nm}$ dimer with a gap of $0.7 \mathrm{~nm}$. d-f) Plots of the LSPR shift $\Delta \lambda$ for monomer (d), dimer transversal mode (e) and longitudinal mode (f) as a function of either the pyridine concentration or the number of adsorbed molecules as calculated by FDTD. 
superimposed on the experimental results, which are also plotted. In figure $4 \mathrm{~d}-\mathrm{f}$, the double Langmuir fit is deconvoluted into two simple Langmuir isotherms (dashed lines). A nice agreement between data and simulations is found on the right part of the plot, confirming that the red shift of the resonance in Region 2 is due to the change of the refractive index around the GNP. The number of molecules required to form the first plateau corresponds to the number of molecules required to create a first monolayer of pyridine. However, on the lower concentration region (spanning from $10^{-15}$ to $10^{-11} \mathrm{~mol}^{-1}$ ) a clear discrepancy appears between experimental data and FDTD. This indicates that the resonance shift is not solely driven by a simple refractive index change, but that another mechanism is involved. We propose charge transfer between pyridine and gold as the main mechanism to explain the resonance shift in Region 1. Such phenomenon has been observed by Goldmann et al. for a monolayer of thiolated aromatic molecules bound to gold nanoparticles. Delocalized electrons from each aromatic molecule are transferred to the GNP. The delocalized electrons from the aromatic ring then take part in the plasmonic resonance, yielding to a modification of the Drude plasma frequency and a significant red shift of its position. ${ }^{24}$

Based on these elements, we can attribute the two constants $K_{1}$ and $K_{2}$ to the pyridinegold (first monolayer) and pyridine-pyridine surface-confined thermodynamic affinity constants, respectively. It is worth noticing that a similar two plateaus behavior with similar values for the affinity constants has been observed by Haes et al, using a different plasmonic platform to detect antigen-antibody interactions. ${ }^{20}$ In our experiments, we clearly observe that the three constants $K$ are almost identical in Region $2\left(K_{2}\right)$, showing that we are in presence of a unique phenomenon i.e. pyridine-pyridine bonding leading to effective refractive index change. In contrast, the constants are different in Region $1\left(K_{1}\right)$, especially for longitudinal mode of the dimer indicating a different behavior in the charge transfer between pyridine and GNP. In particular, a closer look at Figure 4f shows that the first plateau appears for a lower number of molecules than the number required to form a monolayer around the two GNPs ( 10000 molecules, while $\sim 100000$ are necessary for a monolayer $)$. This 
can be understood by noticing that the longitudinal (bonding) mode of a dimer exhibits a strong field enhancement inside the small gap separating the two GNPs, and a much smaller field enhancement from either side of the dimer. As a consequence, the main sensing volume is located in the gap region. ${ }^{25}$ This creates different sensing volumes with different saturation thresholds. Hence, the first kink around $\sim 10000$ molecules can be associated to the saturation of the gap volume, while the full saturation is only reached for $\sim 100000$ molecules.

\section{Performances of the sensor}

In order to compare the performances of our sensor with other designs, it is necessary to introduce a relevant figure of merit. The most common ones for plasmonic sensors are the sensitivity $S$ to the refractive index change (in nm.RIU ${ }^{-1}$ ) and the limit of detection (LoD), i.e. the minimum amount of analyte that can be detected. However, there is no consensus on the best quantity to measure this "minimum amount of analyte": analyte concentration $\left(\mathrm{mol}^{-1}\right)$, number of molecules and mass have already been used. The number of molecules can be misleading, as label-free sensors claiming to reach the single molecule limit often detect huge molecules, such as proteins, weighting several tens of kDa. ${ }^{26}$ Using concentration can also be misleading, as some designs allows to artificially concentrate the initial solution into specific detection areas ${ }^{14}$. The detected mass appears as a more general metric allowing the comparison between different analytes and different kind of sensors.

Table 2: Performance metrics calculated or reported for our sensors and for two SPR sensors from the literature. Values with a $\sim$ sign are estimates.

\begin{tabular}{|c|c|c|c|c|c|}
\hline & Monomer & $\begin{array}{l}\text { Dimer } \\
\text { transversal }\end{array}$ & $\begin{array}{l}\text { Dimer } \\
\text { longitudinal }\end{array}$ & $\begin{array}{l}\text { SPR } \\
27\end{array}$ & $\begin{array}{l}\text { SPR } \\
28\end{array}$ \\
\hline$S(\mathrm{~nm} / \mathrm{RIU})$ & 168 & 171 & 221 & 32000 & $\sim 2000$ \\
\hline FOM & 1.3 & 2.2 & 2.8 & 330 & 50 \\
\hline LoD (\# of molecules) & 1000 & 5000 & 30 & - & - \\
\hline LoD (mass) & $131 \mathrm{zg}$ & $655 \mathrm{zg}$ & $4 \mathrm{zg}$ & $\sim 10 \mathrm{ng}$ & $200 \mathrm{ag}$ \\
\cline { 2 - 6 } & $79 \mathrm{kDa}$ & $395 \mathrm{kDa}$ & $2.4 \mathrm{kDa}$ & $6 \times 10^{12} \mathrm{kDa}$ & $10^{5} \mathrm{kDa}$ \\
\hline
\end{tabular}


Table 2 summarizes several figures of merit for our hybrid photonic-plasmonic nanosensor and from the literature. As references, we have chosen a SPR sensor based on a metamaterial exhibiting one of the best sensitivities reported to date ${ }^{27}$ and another design based on plasmonic nanocavities. ${ }^{28}$ The sensitivity of our sensor has been directly calculated by doing the ratio between the LSPR shift and the refractive index shift. As it is generally the case for LSPR sensors, the measured sensitivity of our sensors $(168<S<221)$ is significantly lower than the sensitivity of SPR sensors $(2000<S<32000)$. To complete this quantity, the FOM (for figure of merit) is often introduced as the ratio between $S$ and the full width at half maximum (FWHM) ${ }^{29}$ We observe again better performances for SPR sensors. However, this two quantities do not reflect all the capabilities of a given molecular sensor.

The main occulted parameter here is the quantity of probed molecules. This quantity is directly dependent on the sensing volume, as probed by the effective volume of the plasmon mode. This sensing volume is around 200 times lower for LSPR than for SPR, ${ }^{30}$ leading to a smaller amount of detected molecules. This amount can be express as a mass, to account for the very different sizes of the molecules targeted by plasmonic sensors, ranging from small molecules to proteins or antibodies. The minimum masses (i.e. the LoD expressed as a mass) that can be detected by our hybrid nanosensor, as measured from Figure 4d-f, are reported in Table 2 and compared with values estimated from the literature. Considering this figure of merit, it is clear that our sensor outperforms the best SPR sensors by several orders of magnitude. The longitudinal resonance of the dimer nanosensor exhibits a LoD as low as 4 zeptograms ( $\mathrm{zg}, 1 \mathrm{zg}=10^{-21} \mathrm{~g}$ ), which is to the best of our knowledge the lowest value ever reported for a plasmonic sensor. The sensor also outperforms biosensors relying on electrical detection, such as semiconducting nanowires ${ }^{31}$ or metallic nanogaps. ${ }^{32}$ In order to find a similar sensing mass performance, one has to turn towards the so-called molecular weighing scales. This is discussed in the following section. 


\section{Plasmonic sensors as molecular weighing scales}

An ideal molecular weighing scale would be a mass sensor capable of weighing minute amounts of matter, down to a single molecule. The most sensitive mass sensors reported to date are based on nanomechanical oscillators: mass sensing of $7 \mathrm{zg}$ has been demonstrated using a microcantilever ${ }^{33}$ and more recently a yoctogram resolution has been demonstrated using an oscillating carbon nanotube as the sensing unit. ${ }^{34}$ However, these impressive results are based on delicate measurements performed both in high vacuum and at cryogenic

temperatures. Measurements of mass in fluids have also been reported, ${ }^{35}$ but with a much lower limit of detection (about $1 \mathrm{fg}$ ). With a LoD in the zeptogram range, our much simpler design appears to be competitive with state-of-the-art mechanical sensors.

An interesting comparison can be made between optical-based and mechanical mass sensors. Actually, our concept can be seen as the optical analog of the mechanical mass sensors: instead of the resonance frequency of a mechanical oscillator, we monitor the resonance frequency of a charge oscillation, i.e., the LSPR sustained by the gold nanoparticle. This optical mass sensor works at room temperature and atmospheric pressure, and enables the detection of mass inside a fluid with a limit of detection at $4 \mathrm{zg}$. We emphasize that within our instrumental limitations, the spectral resolution was $\pm 3 \mathrm{~nm}$. It could be improved by using a brighter source, a more resolved spectrograph and a more sensitive detector. Improving the resolution to $\pm 1 \mathrm{~nm}$ would yield to a limit of detection of $\sim 10$ molecules, corresponding to $\sim 1 \mathrm{zg}$.

\section{Practical issues}

To conclude this discussion, we would like to address the question of the practical use of the hybrid nanosensor. Our fabrication procedure leads to structures that exhibit different geometries (number of GNPs, gap width...) from one micropyramid to another. As the sensing performances are dependent upon the tranduscer's geometry, this creates performance variability from site to site. This implies that prior to any sensing experiment, each 
micropyramid must be optically characterized using polarization-resolved extinction spectroscopy. The resulting extinction spectrum yields the nature of the nanostructure (i.e. the number of grafted GNPs) and their organization. Dimers can be easily identified by their two resonance peaks (also giving the gap width), and the dimer orientation can be resolved by rotation of the polarization axis. It is hence possible to select the most relevant hybrid nanosensor based on the desired sensing parameters (sensitivity and detection range).

It should also be noted that the sensor can be cleaned from any absorbed molecule and reused. We performed on a used sample a cleaning procedure using an oxygen plasma. After the cleaning procedure, we measured the extinction spectrum of the hybrid nanosensor and retrieved the initial position of the plasmon resonance.

\section{Conclusion}

To sum up, we designed, fabricated and characterized a hybrid photonic-plasmonic nanosensor based on the use of quasi-axicon microstructures as optical antennas. Using pyridine as a target molecule, we measured its optical response and evidenced a double Langmuir behavior arising from two contributions. At very low concentrations, the charge transfer between the adsorbed pyridine molecule and the GNP leads to a first resonance shift. This effect is prominent until the first monolayer forms around the plasmonic transducer. After formation of the first monolayer, the accumulation of matter around the transducer starts to increase significantly the effective refractive index around the sensor, shifting the resonance accordingly. With the help of numerical simulations, we were able to estimate the number of molecules adsorbed onto the sensor and hence the corresponding mass. A limit of detection of $4 \mathrm{zg}$ was observed using the longitudinal mode of a dimer of gold nanoparticles. To the best of our knowledge, this value is the lowest amount of matter ever detected using a plasmonic platform and is comparable to state-of-the-art mechanical mass sensors. This device can hence be seen as an optical molecular weighing scale. 
Furthermore, our approach is based on simple nanofabrication processes (optical lithography, chemical etching), which are easily up-scalable and compatible with parallel reading for high-throughput screening. The hybrid sensor shown here is built on a glass substrate, allowing the generation of quasi-Bessel beams only in air. ${ }^{10}$ However, our fabrication process is transferable to higher index transparent materials such as titanium dioxide. Such a structure would be compatible with real-time sensing measurements inside a flow of fluid.

\section{Experimental section}

\section{Nanosensor fabrication}

The array of hybrid photonic-plasmonic nanosensors is made by the following method. ${ }^{10,36}$

(i) A glass coverslip is cleaned using a piranha solution $\left(\mathrm{H}_{2} \mathrm{SO}_{4}: \mathrm{H}_{2} \mathrm{O}_{2}, 1: 3\right)$ in order to create hydroxide groups at the glass surface.

(ii) A monolayer of aminopropyltrimethoxysilane (APTMS) is then grafted on the surface in anhydrous toluene under an argon atmosphere.

(iii) Gold nanoparticles with a $50 \pm 5 \mathrm{~nm}$ diameter are chemically synthesized using the method described by Murphy. ${ }^{37}$ Then, they are grafted onto the APTMS layer by amine-citrate-gold links.

(iv) This grafted GNP monolayer is covered by a positive photoresist (S1813 photoresist, thickness $1.1 \mu \mathrm{m})$. Then the sample is baked at $115{ }^{\circ} \mathrm{C}$ during $90 \mathrm{~s}$ to evaporate the residual solvent and relax residual stress.

(v) The sample is irradiated by a holographic pattern created through the interference of two beams. The period of the pattern is tunable between 1 and $10 \mu \mathrm{m}$. Typically, the chosen period is $4 \mu \mathrm{m}$. The beam used is the $458 \mathrm{~nm}$ line of an argon laser, the power is $3.5 \mathrm{~mW}$ and the irradiation time is $150 \mathrm{~s}$. Then, the sample is rotated by $90^{\circ}$ and 
irradiated under the same conditions in order to obtain a chessboard pattern. Square holes appear in the resist after development.

(vi) A buffered hydrofluoric acid isotropic etching step creates an array of pyramids in the substrate. The residual photoresist protects the GNPs located at their apex. With an optimized etching time, well-defined micropyramids with few GNP sitting at their apex are obtained.

(vii) Finally, the photoresist is lifted-off using acetone, followed by final cleaning step (IPA, water).

\section{Optical setup and measurements}

Extinction spectra have been measured using a home made transmission microscope, coupled to a spectrometer by a multimodal optical fiber. The excitation is performed with a slightly focused (Numerical Aperture $\mathrm{NA}=0.2$ ) halogen lamp. The transmitted light is collected by a x100 air objective lens $(\mathrm{NA}=0.9)$ coupled to a tube lens with a focal length of $150 \mathrm{~mm}$, conducting to a x83 magnification ratio. Spatial filtering of the transmitted light is ensured by a multimodal fiber $(62.5 \mu \mathrm{m}$ core $)$ acting as a confocal pinhole. This yields to a collection spot diameter smaller than $1 \mu \mathrm{m}$, allowing a single hybrid nanosensor measurement. The light is finally analyzed with a monochromator coupled to a CCD camera.

A background $(B k g d)$ measurement is acquired without any excitation light. For each measurement, a reference $I_{0}$ is first taken on a closely located quasi-axicon lens without any GNP on its top. Using a close position for the reference allows us to assume that both lenses experienced the same etching conditions, leading to similar shapes and hence optical properties. The signal $I$ is then collected from the transducer. We typically averaged 10 spectra with an acquisition of $2 \mathrm{~s}$ each. The extinction spectra is calculated using the usual formula: 


$$
Q_{e x t}=-\log \left(\frac{I-B k g d}{I_{0}-B k g d}\right)
$$

A Savitzky-Golay smoothing process (second degree polynomial function over 51 points) is performed on the spectra to decrease the measurement uncertainty to $\pm 3 \mathrm{~nm}$.

\section{Acknowledgement}

We thank Hélène Yockell-Lelièvre for DDA calculations and Claire Deeb for AFM imaging. This work has been supported by the EIPHI Graduate School (contract "ANR-17EURE-0002"). Financial support of Nano'Mat (www.nanomat.eu) by the "Ministère de l'enseignement supérieur et de la recherche", the "Conseil régional Champagne-Ardenne", the "FEDER fund" and the "Conseil général de l'Aube" is acknowledged. We also acknowledge support from the CNRS and the Agence Nationale de la Recherche (ANR) under HYNNA, TWINS and NATO grants.

\section{Supporting Information Available}

The following files are available free of charge.

- Figure S1: Morphological characterization of the hybrid sensor

- Figure S2: Signal to noise ratio calculation

- Figure S3: Pyridine stacking around a GNP

\section{References}

1. Anker, J. N.; Hall, W. P.; Lyandres, O.; Shah, N. C.; Zhao, J.; Van Duyne, R. P. Biosensing with plasmonic nanosensors. Nat. Mater. 2008, 7, 442-453. 
2. Estevez, M. C.; Otte, M. A.; Sepulveda, B.; Lechuga, L. M. Trends and challenges of refractometric nanoplasmonic biosensors: A review. Analytica Chimica Acta 2014, 806, $55-73$.

3. Tokel, O.; Inci, F.; Demirci, U. Advances in Plasmonic Technologies for Point of Care Applications. Chem. Rev. 2014, 114, 5728-5752.

4. Masson, J.-F. Surface Plasmon Resonance Clinical Biosensors for Medical Diagnostics. ACS Sensors 2017, 2, 16-30.

5. Mayer, K. M.; Hafner, J. H. Localized Surface Plasmon Resonance Sensors. Chem. Rev. 2011, 111, 3828-3857.

6. Yavas, O.; Aćimović, S. S.; Garcia-Guirado, J.; Berthelot, J.; Dobosz, P.; Sanz, V.; Quidant, R. Self-Calibrating On-Chip Localized Surface Plasmon Resonance Sensing for Quantitative and Multiplexed Detection of Cancer Markers in Human Serum. ACS Sensors 2018, 3, 1376-1384.

7. Novotny, L.; Van Hulst, N. Antennas for light. Nat. Photon. 2011, 5, 83-90.

8. Regmi, R.; Berthelot, J.; Winkler, P. M.; Mivelle, M.; Proust, J.; Bedu, F.; Ozerov, I.; Begou, T.; Lumeau, J.; Rigneault, H. et al. All-Dielectric Silicon Nanogap Antennas to Enhance the Fluorescence of Single Molecules. Nano Lett. 2016, 16, 5143-5151.

9. Gérard, D.; Devilez, A.; Aouani, H.; Stout, B.; Bonod, N.; Wenger, J.; Popov, E.; Rigneault, H. Efficient excitation and collection of single-molecule fluorescence close to a dielectric microsphere. J. Opt. Soc. Am. B 2009, 26, 1473-1478.

10. Martin, J.; Proust, J.; Gérard, D.; Bijeon, J.-L.; Plain, J. Intense Bessel-like beams arising from pyramid-shaped microtips. Opt. Lett. 2012, 37, 1274-1276.

11. Lindfors, K.; Kalkbrenner, T.; Stoller, P.; Sandoghdar, V. Detection and spectroscopy 
of gold nanoparticles using supercontinuum white light confocal microscopy. Phys. Rev. Lett. 2004, 93, 037401.

12. Gandubert, V. J.; Lennox, R. B. Assessment of 4-(dimethylamino) pyridine as a capping agent for gold nanoparticles. Langmuir 2005, 21, 6532-6539.

13. Larson, I.; Chan, D.; Drummond, C. J.; Grieser, F. Use of atomic force microscopy force measurements to monitor citrate displacement by amines on gold in aqueous solution. Langmuir 1997, 13, 2429-2431.

14. De Angelis, F.; Gentile, F.; Mecarini, F.; Das, G.; Moretti, M.; Candeloro, P.; Coluccio, M. L.; Cojoc, G.; Accardo, A.; Liberale, C. et al. Breaking the diffusion limit with super-hydrophobic delivery of molecules to plasmonic nanofocusing SERS structures. Nat. Photon. 2011, 5, 682-687.

15. Zayats, A. V.; Smolyaninov, I. I.; Maradudin, A. A. Nano-optics of surface plasmon polaritons. Phys. Rep. 2005, 408, 131-314.

16. Marcus, Y. The properties of solvents; Wiley, 1998.

17. You, N.-H.; Suzuki, Y.; Yorifuji, D.; Ando, S.; Ueda, M. Synthesis of high refractive index polyimides derived from 1, 6-bis (p-aminophenylsulfanyl)-3, 4, 8, 9-tetrahydro2, 5, 7, 10-tetrathiaanthracene and aromatic dianhydrides. Macromolecules 2008, 41, 6361-6366.

18. Riboh, J. C.; Haes, A. J.; McFarland, A. D.; Ranjit Yonzon, C.; Van Duyne, R. P. A nanoscale optical biosensor: real-time immunoassay in physiological buffer enabled by improved nanoparticle adhesion. J. Phys. Chem. B 2003, 10\%, 1772-1780.

19. Haes, A. J.; Hall, W. P.; Chang, L.; Klein, W. L.; Van Duyne, R. P. A localized surface plasmon resonance biosensor: First steps toward an assay for Alzheimer's disease. Nano Lett. 2004, 4, 1029-1034. 
20. Haes, A. J.; Chang, L.; Klein, W. L.; Van Duyne, R. P. Detection of a biomarker for Alzheimer's disease from synthetic and clinical samples using a nanoscale optical biosensor. J. Am. Chem. Soc. 2005, 127, 2264-2271.

21. Mootz, D.; Wussow, H.-G. Crystal structures of pyridine and pyridine trihydrate. J. Chem. Phys. 1981, 75, 1517-1522.

22. de Oteyza, D. G.; Gorman, P.; Chen, Y.-C.; Wickenburg, S.; Riss, A.; Mowbray, D. J.; Etkin, G.; Pedramrazi, Z.; Tsai, H.-Z.; Rubio, A. et al. Direct imaging of covalent bond structure in single-molecule chemical reactions. Science 2013, 340, 1434-1437.

23. Deeb, C.; Zhou, X.; Plain, J.; Wiederrecht, G. P.; Bachelot, R.; Russell, M.; Jain, P. K. Size Dependence of the Plasmonic Near-Field Measured via Single-Nanoparticle Photoimaging. J. Phys. Chem. C 2013, 117, 10669-10676.

24. Goldmann, C.; Lazzari, R.; Paquez, X.; Boissière, C.; Ribot, F.; Sanchez, C.; Chanéac, C.; Portehault, D. Charge Transfer at Hybrid Interfaces: Plasmonics of Aromatic Thiol-Capped Gold Nanoparticles. ACS Nano 2015, 9, 7572-7582.

25. Acimovic, S. S.; Kreuzer, M. P.; González, M. U.; Quidant, R. Plasmon near-field coupling in metal dimers as a step toward single-molecule sensing. ACS Nano 2009, 3, $1231-1237$.

26. Taylor, A. B.; Zijlstra, P. Single-Molecule Plasmon Sensing: Current Status and Future Prospects. ACS Sensors 2017, 2, 1103-1122.

27. Kabashin, A.; Evans, P.; Pastkovsky, S.; Hendren, W.; Wurtz, G.; Atkinson, R.; Pollard, R.; Podolskiy, V.; Zayats, A. Plasmonic nanorod metamaterials for biosensing. Nat. Mater. 2009, 8, 867-871.

28. Dell'Olio, F.; Conteduca, D.; De Palo, M.; Ciminelli, C. Design of a New Ultracompact 
Resonant Plasmonic Multi-Analyte Label-Free Biosensing Platform. Sensors 2017, 17, doi: $10.3390 / \mathrm{s} 17081810$.

29. Scheinfein, M.; Muray, A.; Isaacson, M. Electron energy loss spectroscopy across a metalinsulator interface at sub-nanometer spatial resolution. Ultramicroscopy 1985, 16, 233239.

30. Zlatanovic, S.; Mirkarimi, L. W.; Sigalas, M. M.; Bynum, M. A.; Chow, E.; Robotti, K. M.; Burr, G. W.; Esener, S.; Grot, A. Photonic crystal microcavity sensor for ultracompact monitoring of reaction kinetics and protein concentration. Sensors and Actuators B: Chemical 2009, 141, 13-19.

31. Stern, E.; Klemic, J. F.; Routenberg, D. A.; Wyrembak, P. N.; Turner-Evans, D. B.; Hamilton, A. D.; LaVan, D. A.; Fahmy, T. M.; Reed, M. A. Label-free immunodetection with CMOS-compatible semiconducting nanowires. Nature 2007, 445, 519.

32. Kim, S. K.; Cho, H.; Park, H.-J.; Kwon, D.; Lee, J. M.; Chung, B. H. Nanogap biosensors for electrical and label-free detection of biomolecular interactions. Nanotechnology 2009, 20, 455502 .

33. Yang, Y. T.; Callegari, C.; Feng, X. L.; Ekinci, K. L.; Roukes, M. L. Zeptogram-Scale Nanomechanical Mass Sensing. Nano Lett. 2006, 6, 583-586.

34. Chaste, J.; Eichler, A.; Moser, J.; Ceballos, G.; Rurali, R.; Bachtold, A. A nanomechanical mass sensor with yoctogram resolution. Nat. Nanotechnol. 2012, 7, 301-304.

35. Burg, T. P.; Godin, M.; Knudsen, S. M.; Shen, W.; Carlson, G.; Foster, J. S.; Babcock, K.; Manalis, S. R. Weighing of biomolecules, single cells and single nanoparticles in fluid. Nature 2007, 446, 1066-1069.

36. Barbillon, G.; Bijeon, J.-L.; Lérondel, G.; Plain, J.; Royer, P. Detection of chemical molecules with integrated plasmonic glass nanotips. Surf. Sci. 2008, 602, L119-L122. 
37. Jana, N. R.; Gearheart, L.; Murphy, C. J. Evidence for Seed-Mediated Nucleation in the Chemical Reduction of Gold Salts to Gold Nanoparticles. Chem. Mater. 2001, 13, 2313-2322. 


\section{Graphical TOC Entry}

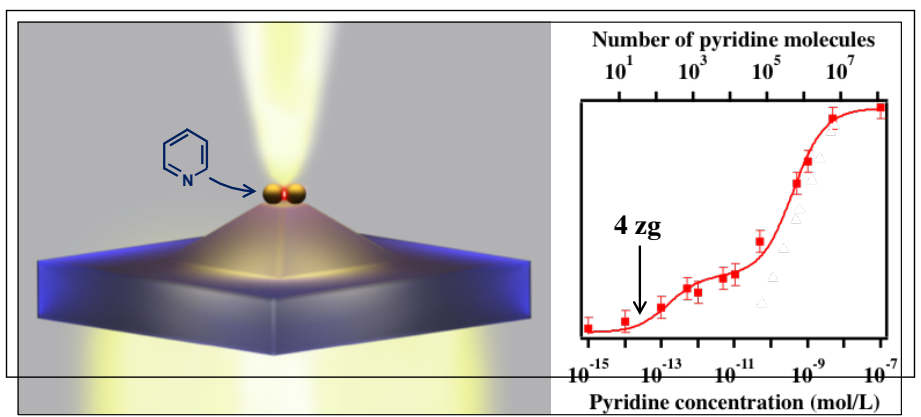

